\title{
La gentrification rurale d'une Area of Outstanding Natural Beauty (AONB) : maitrise et instrumentalisations du planning system anglais dans les Cotswolds
}

Rural gentrification in an Area of Outstanding Natural Beauty (AONB): control and use of the planning system in the Cotswolds

Ländliche Gentrifizierung in einem Gebiet von außergewöhnlicher natürlicher Schönheit (AONB): Kontrolle und Nutzung des Planungssystems in den Cotswolds

\section{Marie Méténier}

\section{OpenEdition} Journals

Édition électronique

URL : http://journals.openedition.org/rge/7976

DOI : $10.4000 /$ rge. 7976

ISSN : 2108-6478

\section{Éditeur}

Association des géographes de l'Est

\section{Référence électronique}

Marie Méténier, «La gentrification rurale d'une Area of Outstanding Natural Beauty (AONB) : maitrise et instrumentalisations du planning system anglais dans les Cotswolds », Revue Géographique de l'Est [En ligne], vol. 58 / 1-2 | 2018, mis en ligne le 19 avril 2019, consulté le 08 septembre 2020. URL : http://journals.openedition.org/rge/7976 ; DOI : https://doi.org/10.4000/rge.7976 


\section{La gentrification rurale d'une Area of Outstanding Natural Beauty (AONB) : maitrise et instrumentalisations du planning system anglais dans les Cotswolds}

Rural gentrification in an Area of Outstanding Natural Beauty (AONB): control and use of the planning system in the Cotswolds

Ländliche Gentrifizierung in einem Gebiet von außergewöhnlicher natürlicher Schönheit (AONB): Kontrolle und Nutzung des Planungssystems in den Cotswolds

Marie Méténier

\section{Introduction :}

1 Depuis la fin des années 1970, la gentrification rurale a fait l'objet de nombreuses recherches en Angleterre (Parsons, 1979,1980 ; Cloke, 1979, 1983 ; Little, 1987 ; Phillips, 1993 ; Cloke et al., 1995 ; Smith, 1998) et plus récemment (Smith et Phillips, 2001 ; Phillips, 2010 ; Richard, 2009). Si les espaces ruraux anglais ont été largement explorés par les chercheurs, les espaces «naturels» protégés ont été marginalisés dans la littérature (Richard, 2017). Pourtant, étudier le processus de gentrification rurale dans ces espaces est pertinent dans la mesure où le Royaume-Uni est un pays densément peuplé (Richard, 2009) ce qui conduit à rendre les espaces "naturels» extrêmement attractifs pour des «middle classes " à la recherche d'un cadre de vie riche d'aménités environnementales (Marsden et al., 1996; Murdoch, Lowe, 2003). De plus, les espaces protégés anglais ont été depuis longtemps habités et façonnés par l'homme (Blunden, Curry, 1992 ; MacEwen, MacEwen, 1989) et sont particulièrement idéalisés et investis de forts sentiments d'appartenance identitaire (Bailoni, 2012). Par conséquent, le fait de 
conférer à certains de ces espaces une protection au titre du droit de l'environnement n'a fait qu'accentuer cette attraction ce qui a généré l'apparition de conflits et d'instrumentalisations situés à l'interface géolégale, au cœur du planning system anglais. D.Parsons $(1979,1980)$ et P.Cloke $(1979,1983)$, les co-fondateurs du concept de gentrification rurale, avaient dénoncé l'articulation du planning system anglais avec le processus de gentrification rurale. Plus particulièrement, les chercheurs ont analysé les conséquences sociales de politiques publiques comme la "key settlement policy" introduite par le Town and Country Planning Act de 1968. Comme le titre de la politique le suggère, la philosophie suivie par les aménageurs anglais consistait à imposer des restrictions urbanistiques extrêmement strictes dans les villages qui n'étaient pas identifiés pour regrouper les services et les investissements publics plutôt que de risque d'entrainer la destruction d'espaces susceptibles de présenter une valeur (environnementale et économique) ultérieure. Un déplacement-remplacement s'est opéré entre les ménages ruraux modestes qui ont été incités à rejoindre les «key settlements " villages, où se concentraient les services et la modernité d'après-guerre, libérant alors une grande partie du parc immobilier dans les espaces ruraux. Ces derniers ont été progressivement investis par des ménages plus aisés, plus mobiles et le plus souvent urbains, portés par un idéal de vie dans un environnement sain, esthétique et protégé.

2 En s'appuyant sur la définition "souple " de la gentrification proposée par E.Clark (2005), l'objectif de cet article est de comprendre comment le planning system anglais peut être mobilisé et instrumentalisé par des "gentrifieurs" au sein d'un cadre territorial spécifique que sont les Area of Outstanding Natural Beauty (AONBs).

3 La méthodologie employée pour cet article s'inscrit dans une démarche géo-légale (Garcier, 2009) et repose sur une enquête qualitative basée sur un corpus de 80 entretiens semi-directifs réalisés sur une période de quatre mois de 2015 à 2018. Etudier les espaces protégés en cours de gentrification ou gentrifiés permet de mobiliser non seulement la géographie rurale, la géographie de l'environnement, mais aussi le droit de l'environnement et le droit de l'urbanisme regroupés et articulés dans le planning system anglais. La mobilisation de disciplines complémentaires pour traiter un même sujet s'inscrit dans une approche interdisciplinaire propre à la géographie du droit (Forest, 2009). Comme le souligne S. Depraz (2008), l'espace protégé constitue « un objet géographique à part entière ». Il est également un objet "norme » intrinsèquement lié à l'ensemble des règles juridiques consécutives à son classement qui encadrent et règlementent les usages dans le périmètre de protection déterminé. Dans le contexte anglais, l'emploi des guillemets concernant ces espaces "naturels» se justifie dans la mesure où la protection qui leur est conférée témoigne d'une forme d'artificialisation supplémentaire justifiée par une pression anthropique locale exacerbée (Depraz, 2008). En tant qu'objets géo-légaux, les espaces protégés sont donc particulièrement intéressants à analyser à la lumière de la géographie du droit.

Pour contextualiser l'enquête empirique, cette dernière a été menée dans un espace protégé anglais, l'Area of Outstanding Natural Beauty (AONB) des Cotswolds. Comme le suggère R.Platt (2004), la géographie du droit permet d'introduire les terrains de recherche d'une manière pertinente et complémentaire. Géographiquement, l'AONB des Cotswolds, située au sud-est de l'Angleterre, forme un ensemble de cuestas qui bénéficient d'une renommée liée à l'image d'Angleterre quintessentielle qui leur est attribuée. Constitués de collines ondoyantes au sein desquelles serpentent rivières et 
ruisseaux, les Cotswolds se caractérisent surtout pour leur architecture vernaculaire dont les roches, couleurs de miels, ont fait la réputation des cottages anglais, symbole de l'idylle rurale que les Anglais entretiennent avec leur campagne (Woods, 2017). Quelques études sur la gentrification rurale ont rapidement démontré le potentiel des Cotswolds en tant qu'espace sujet au processus de gentrification rurale (Cloke et al., 1995; Schmied, 2002; Richard, 2017) sans pour autant s'arrêter sur le rôle du mécanisme de protection qui leur est conféré. Les Cotswolds sont en effet idéalement situés par rapport à des villes comme Bristol, Swindon, Oxford et plus encore, très facilement accessibles depuis Londres (figure 1).

Figure 1 : L'AONB des Cotswolds : localisation géographique, pressions et flux sur un espace protégé anglais (Marie Méténier, 2018).

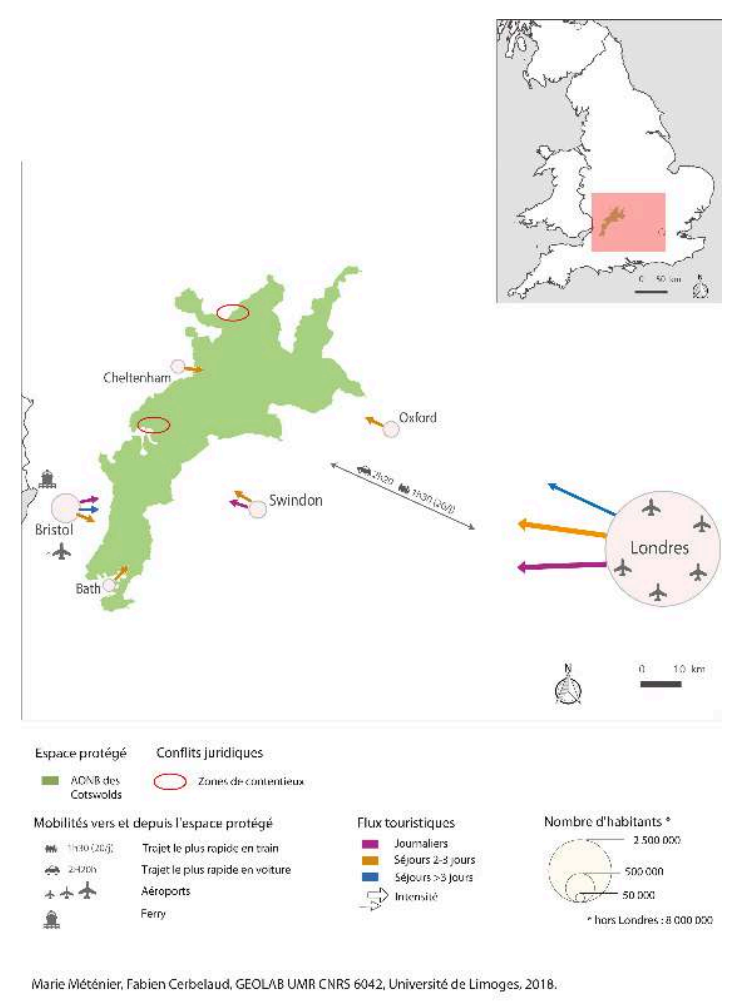

Dans l'AONB des Cotswolds, la moyenne du prix d'un logement en 2011 était de £362 200 par comparaison à une moyenne nationale de £159 385 (Cumulus Consultant Ltd.2013). Ce différentiel de prix et l'attractivité résidentielle pour l'AONB des Cotswolds a conduit à intensifier la problématique de logements financièrement accessibles pour les populations rurales aux revenus modestes qui ont progressivement été remplacées et déplacées des villages ruraux (Marsden et al., 1996). Venant se superposer à ces caractéristiques géographiques, les Cotswolds ont été désignés AONB en 1966. Ne correspondant à aucune limite administrative connue, elle est la plus vaste AONB du territoire britannique, car elle représente près de $10 \%$ de la superficie totale de l'ensemble des AONBs. Le mécanisme des AONB a été introduit suite à l'entrée en vigueur du «National Parks and Access to the Countryside Act» en 1949. L'intitulé de ce texte est éloquent au regard de la distinction opérée entre les parcs nationaux et les AONB, la différence fondamentale résidant dans le niveau de protection et les compétences juridiques conférées aux gestionnaires de ces espaces. Les premiers parcs nationaux anglais ont été désignés en 1951 et disposent, depuis l'entrée en vigueur de l' 
Environment Act de 1995, de compétences élargies en matière d'urbanisme puisqu'elles en constituent les autorités compétentes pour la superficie du parc (une compétence qui était auparavant détenue par les autorités locales). De leur côté, les AONBs ont pendant longtemps été considérées comme les «parents pauvres» des espaces protégés britanniques (Holdaway, Smart, 1999). En effet, les AONBs n'ont été désignées qu'une fois la première vague de création des parcs nationaux anglais aboutie (la première AONB étant celle de la péninsule de Gower désignée en 1956 au Pays-deGalles) et les moyens financiers et humains qui leur ont été attribués ont pendant longtemps été jugés comme négligeables par comparaison aux parcs nationaux (MacEwen, MacEwen, 1989). Ce n'est qu'en 2000 que le « Countryside Right of Way Act » a permis de faire évoluer les statuts, les compétences et les moyens attribués aux AONBs. Il est désormais possible pour les AONBs de créer un "conservation board", autorité indépendante calquée sur le modèle des "national parks authorities». Néanmoins, la compétence urbanistique ne leur a pas été transférée et reste à la charge des autorités locales. Le "conservation board» des Cotswolds a été officiellement créé en 2005, fait relativement rare puisque seulement deux des 38 AONBs désignées en Angleterre en bénéficient.

6 Incarnant le principe de territorialisation du droit en raison de sa double identité (Depraz, 2008), l'espace protégé peut être défini à la fois comme un objet «norme » et comme un objet «espace». Cela permet de justifier l'analyse du processus de gentrification rurale pour démontrer de quelle manière l'AONB des Cotswolds peut être appropriée et modelée à différentes échelles (I). L'espace protégé est également un facteur d'influence du droit puisque le contexte social et environnemental dans lequel il s'inscrit peut entrainer une modification de l'application des règlementations, voire la production de nouvelles normes, destinées à adapter ou à s'adapter à la gentrification rurale d'un espace protégé. Cependant, les mécanismes de protection peuvent également faire l'objet de contestation, notamment pour ce qui concerne leur emprise spatiale et aboutir in fine à des contentieux (II).

\section{L'AONB des Cotswolds : un espace protégé modelé par la gentrification rurale.}

\section{Le mécanisme de protection «AONB » : critère déterminant ou plus- value dans les stratégies résidentielles?}

7 Le droit est source de spatialité. Par conséquent, il influence les déplacements et les comportements des individus dans l'espace social (Delaney, 2009). Processus de recomposition démographique et social, la gentrification implique une démarche migratoire qui, dans le cadre de la gentrification rurale, peut être qualifiée d'une forme de migration d'aménités (Richard et al., 2014). Ces aménités environnementales présentées par les espaces ruraux attractifs sont fréquemment associées à un cadre de vie idéal, souvent conçu par opposition à une vie en milieu urbain. La majorité des espaces ruraux anglais remplissent de manière plus ou moins aboutie ces critères de vie. En Angleterre, les dynamiques migratoires vers les espaces ruraux et protégés sont anciennes (Newby, 1979; Urry, 1995) et se sont généralisées à partir de la seconde moitié du 20 ème siècle (Woods, 2007). Cette antériorité pourrait en soit légitimement justifier que les chercheurs britanniques sur la gentrification rurale ne se soient 
attaché qu'à décrire les conséquences du processus plutôt que de mettre en exergue les projets migratoires à destination des espaces ruraux (Richard, 2017). Si les chercheurs anglais ne se sont que peu intéressés à cette dimension, il en va autrement des travaux des français et américains qui ont analysé, dans le cadre de leurs recherches sur le renouveau des campagnes post-industrielles des pays occidentaux, les liens entre migrations d'aménités et gentrification rurale (Cognard, 2010 ; Glorioso, Moss, 2012 ; Abrams et al., 2012 ; Nelson et al.,2011).

Dans le cadre d'une recherche sur le processus de gentrification rurale dans un espace protégé anglais, il aurait pu être choisi d'interroger le rôle des aménités environnementales dans les projets migratoires des gentrifieurs. Néanmoins, l'étude spécifique des espaces protégés permettait dès l'élaboration de la grille d'entretiens d'introduire une nouvelle entrée dans la réflexion. En s'intéressant au mécanisme de protection de l'environnement, il s'agissait alors de comprendre si ce dernier était un critère de choix déterminant (ou non) dans les stratégies résidentielles des ménages aisés. En d'autres termes, il s'agissait d'analyser si le fait que les Cotswolds soient désignés en tant qu'AONB, et donc protégés, ait pu être une condition substantielle dans le projet d'installation des gentrifieurs. Pour D. Schmid (2002), la désignation des Cotswolds en tant qu'AONB a sans aucun doute renforcé l'image positive renvoyée par cet espace. Néanmoins, en s'appuyant sur les conclusions présentées par Preece (1981), D.Schmied avait conclu que s'installer dans un espace désigné Area of Outstanding Natural Beauty relevait plus d'une forme de plus-value que d'un réel objectif pour les gentrifieurs (Schmied, 2002). Plusieurs interrogations peuvent être tirées de ces résultats. Tout d'abord le droit, ou plutôt le mécanisme de protection conféré par la loi à un espace, ne serait donc pas source de spatialité ? Un questionnement à relativiser dans la mesure où il ressort des entretiens qu'en amont, la protection juridique conférée aux Cotswolds soit au mieux une plus-value, cela devient tout à fait significatif dès lors qu'il s'agit de lutter contre l'étalement urbain et de protéger l'investissement réalisé dans un cadre environnemental représenté et idéalisé. Ce basculement de simple plus-value d'un périmètre de protection à critère déterminant lorsqu'il s'agit de protéger son cadre de vie fait échos aux analyses réalisées par D.Desponds (2007) sur les parcs naturels régionaux (PNR) franciliens. Le mécanisme de protection joue alors un rôle central dans le renforcement des logiques ségrégatives via l'instrumentalisation des documents d'urbanisme. Cependant, contrairement aux parcs nationaux, le mécanisme des AONB reste un mécanisme peu contraignant juridiquement et peu visible, si bien que certains auteurs les qualifient de "parents-pauvres » des espaces protégés anglais (Holdaway, Smart, 1999). Les entretiens réalisés pour cette recherche révèlent qu'une tendance très nette se dessine. La désignation des Cotswolds en tant qu'AONB n'était en effet considérée que comme une plus-value pour la majorité des ménages installés avant les années 2000. Cependant, pour les ménages installés depuis 2010, le mécanisme de protection constitue un critère déterminant dans leur stratégie résidentielle compte tenu de la pression urbanistique exercée sur l'ensemble des espaces ruraux anglais.

« Nous vivions à proximité de Swindon, dans la campagne. Depuis les élections de Cameron, le gouvernement a lancé un programme massif de nouvelles constructions qui ruinent la campagne. Derrière chez nous, un projet de 200 nouveaux logements a été autorisé. On a donc décidé il y a 4 ans de venir s'installer dans les Cotswolds, à Lower Slaughter, parce que c'est une AONB, donc normalement on devrait être à l'abri ». (Entretien n52, femme, 43 ans, mariée, un enfant, mai 2016) 
Cette volonté de se protéger, de se mettre "à l'abri», se retrouve fréquemment dans les discours, mais se matérialise aussi directement dans la sphère privée et se traduit par la multiplication de marqueurs spatiaux érigés comme de véritables défenses contre les agressions extérieures.

\section{Les marqueurs de la gentrification rurale : propriété privée et signes manifestes d'exclusion.}

10 Nicholas Blomley (2004), en s'appuyant sur la fable pour enfant «Pierre Lapin » écrite par Béatrix Potter, a étudié les limites de la propriété privée dans une perspective géolégale. Son objectif était de montrer l'influence du droit sur le paysage à travers les marqueurs spatiaux que sont par exemple les clôtures ou les signes d'interdiction. Le processus de gentrification rurale, s'il est souvent associé à l'installation dans un environnement présentant de nombreuses aménités, renvoie également à une recherche active d'isolement au sein de la sphère domestique (Cloke, 1983 ; Smith, 1998, Richard et al., 2014). Comme l'illustre la figure 2, l'appropriation de l'espace rural idéalisé se matérialise alors par l'installation de marqueurs spatiaux pouvant être associés au contrôle de l'espace investi (Richard, 2009).

Figure 2 : Formes d'isolement au sein de la sphère domestique et marqueurs spatiaux consécutifs à la gentrification rurale d'un espace protégé (Marie Méténier, avril 2016 et mai 2017).

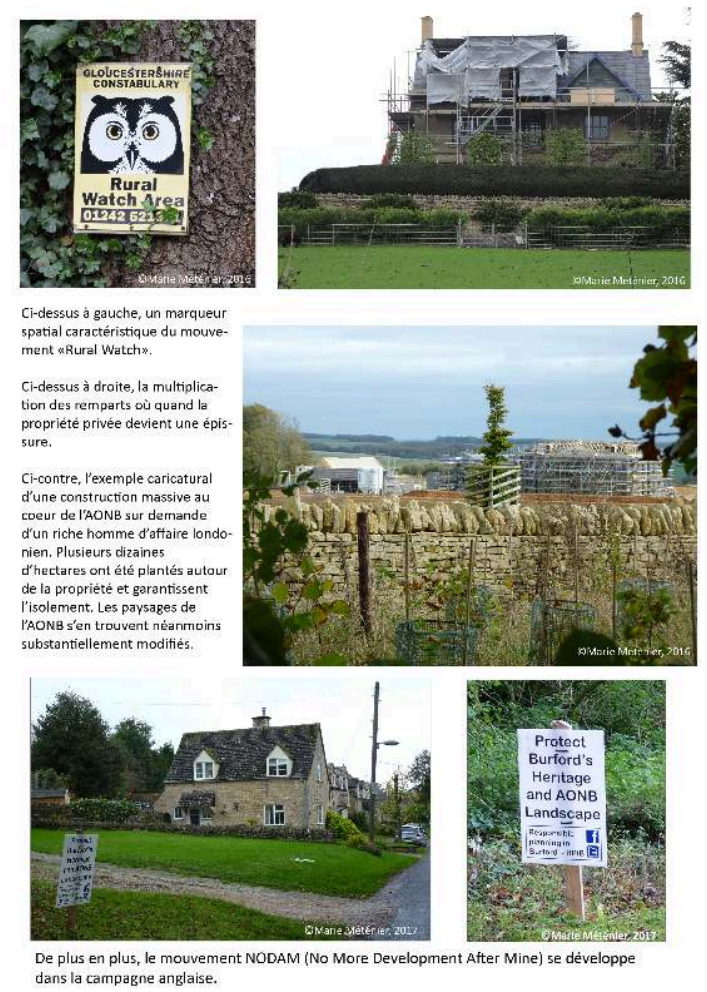

11 Comment dès lors gérer un espace gentrifié dans la mesure où, comme cela a été rappelé en introduction, le Cotswolds Board ne peut intervenir que par l'encadrement des comportements à défaut de pouvoirs coercitifs ? Face à la modification de certaines pratiques pouvant avoir un impact direct sur le paysage de l'espace protégé dont l'autorité gestionnaire est en charge, des adaptations peuvent être apportées à travers 
des documents juridiques plus ou moins contraignants. Il est possible ici de prendre l'exemple de l'adaptation au processus de gentrification d'un document produit par le Cotswolds Board en 2016, intitulé : « Cotswolds Landscape Strategy and Guidelines - revision V4 for adoption March $2016^{1} »$.

12 Comparer ce document avec celui produit en 2008 permet notamment de se rendre compte des impacts de la gentrification sur le paysage et de voir comment l'autorité gestionnaire tente d'y apporter une réponse adaptée, dans la limite des pouvoirs qui lui sont attribués. Ainsi, deux règlementations ont été introduites : la première concerne la vente séparée d'un corps de ferme du reste de l'exploitation agricole tandis que la seconde concerne les espaces boisés et le bocage. L'impact de la gentrification rurale sur le bâti agricole et rural est tangible au regard des multiples rénovations et conversions de bâtiments à destination agricole en logement d'habitation par les ménages aisés venus s'implanter. Cette conversion du bâti, marqueur de la gentrification rurale, a conduit l'autorité gestionnaire de l'AONB des Cotswolds à restreindre et encadrer le changement de destination des bâtiments agricoles. Lorsque cette dernière est sollicitée pour avaliser une demande de conversion d'un bâtiment agricole, sa politique est désormais de rendre un avis négatif. Dans ses documents de planification, elle a introduit des conditions d'urbanisations plus strictes pour restreindre le morcellement des parcelles agricoles et s'oppose de manière systématique à la construction de logements à usage d'habitation, y compris les logements d'habitation pour les exploitants agricoles, dans l' "open countryside" (correspondant aux zones naturelles et agricoles dans le droit de l'urbanisme anglais). Ce faisant, les règlementations introduites par le gestionnaire de l'espace protégé contribuent à renforcer les règles du planning system relatives aux AONB pour que celles-ci se rapprochent du niveau de coercition appliqué dans les parcs nationaux.

Un second exemple, en lien avec le contrôle de la propriété privée, permet d'illustrer la manière dont les gestionnaires d'espaces protégés s'adaptent au processus de gentrification rurale. La quête de l'isolement, souvent matérialisée au niveau de la sphère domestique, impacte de manière récurrente les espaces boisés et le réseau bocager, allant dans les cas les plus extrêmes jusqu'à la création de nouvelles parcelles boisées qui modifient de manière substantielle les paysages de l'AONB. Or le maintien et la conservation des paysages de l'AONB constituent l'objectif statutaire premier de ces espaces. Les gestionnaires de l'AONB utilisent une terminologie précise pour qualifier cette recherche d'isolement à travers le terme de "shelterbelts ", synonyme d'une sorte de ceinture végétale destinée à couper du monde ceux qui les introduisent. Afin de réguler cette conséquence du processus de gentrification rurale, la stratégie adoptée est graduelle. Elle peut aller du simple fait de décourager et limiter les plantations relatives à ces « shelterbelts » jusqu'à requérir la réalisation d'une « Environmental Impact Assessment » (EIA) par la Forestry Commission pour déterminer si une autorisation est nécessaire pour poursuivre le boisement d'une parcelle et auquel cas s'y opposer si cela porte atteinte aux règles fixées pour maintenir le(s) paysage(s) de l'AONB. Par comparaison aux pouvoirs conférés aux gestionnaires des parcs nationaux anglais, ceux conférés aux AONB restent d'une manière générale très limités et consultatifs. Malgré tout, en essayant de contrôler l'impact paysager du processus de gentrification rurale par la mise en œuvre de différentes mesures, les AONBs peuvent être considérées comme des interfaces géo-légales où les règlementations s'adaptent au processus géographique et vice-versa. 


\title{
Renforcer les limites des espaces protégés : l'enjeu de la cristallisation du foncier.
}

\author{
L'exclusion par l'instrumentalisation du planning system : des \\ gentrifieurs NYMBY.
}

Si les clôtures ou les signes d'interdiction sont les éléments le plus souvent visibles de la matérialité du droit, l'influence du droit sur le paysage peut également se présenter de manière immatérielle dans le but de figer un paysage en élargissant le champ d'application d'un outil règlementaire. En l'occurrence, l'instrumentalisation du droit entre directement en relation avec un autre aspect de la gentrification rurale relatif au fait que les gentrifieurs, en tant que néo-ruraux, défendent avec vigueur cette partie de la campagne anglaise dans laquelle ils sont venus s'installer et s'investissent dans la lutte contre l'étalement urbain (Pistre, 2010). Un investissement pouvant parfois être qualifié de NIMBY ${ }^{2}$ pour désigner ce comportement des caractéristiques de certains gentrifieurs (Lowe et Murdoch, 2003). Comme le rappelle M. Bailoni (2012), l'attitude NIMBY illustre l'investissement des populations dans la défense d'un patrimoine culturel et naturel potentiellement menacé par un projet d'aménagement de dimension variable. Les gentrifieurs s'investissent donc au service de la protection d'une qualité de vie, du calme et de la beauté des Cotswolds, mais également dans le but de protéger leur patrimoine immobilier. En effet, le développement de l'urbanisation pourrait, à terme, affecter la valeur marchande de leur bien. Certains n'hésitent donc pas à s'investir dans la vie politique locale (Lowe, 1977) et, non contents des actions et des pouvoirs limités conférés à l'autorité gestionnaire de l'espace protégé pour réguler la pression urbanistique, ils instrumentalisent les outils de planification comme le mécanisme des "conservation areas" afin de protéger leur cadre de vie. Une telle démarche a pu être constatée notamment dans le petit village de Laverton, situé au nord de l'AONB des Cotswolds à la frontière de l'espace protégé. Laverton, tout comme les villages de Buckland et de Stanton contigus à ce premier, fait partie des villages très prisés des Cotswolds. Pourtant la pression urbanistique exercée aux pieds de ces collines est réelle et constitue une source d'inquiétude pour la population interrogée. L'extrait d'entretien réalisé avec couple de banquiers londoniens, installés depuis 2 ans dans le village, et le schéma ci-dessous permettent de résumer la situation conflictuelle où la géographie rencontre le droit :

"Les Cotswolds sont de plus en plus convoités, envahis. Nous avons très peur qu'avec la pression démographique, les Cotswolds ne soient saccagés par les politiques menées en matière de nouvelles constructions qui nous menacent directement. Ici, à Laverton, le centre du village est classé en tant que conservation area. Nous sommes inclus dans l'AONB des Cotswolds, mais la route là-bas constitue la limite. Le Cotswolds Board ne fait rien pour protéger l'environnement, ils ne peuvent pas s'opposer à de nouvelles constructions, ils n'ont aucun pouvoir. Donc, je m'efforce, depuis que je suis élu de faire réviser la "conservation area" pour y inclure les champs qui séparent les trois villages et qui constituent une richesse inestimable pour les villageois » (Entretien n³3, homme, 54 ans, marié, deux enfants, mai 2016).

La figure 3 résume le projet de cet élu. Les trois villages mentionnés sont respectivement séparés par plusieurs parcelles agricoles sur lesquelles les habitants exercent un droit de passage qui relie les villages entre eux. 
Figure 3 : Schéma du projet de création des champs entre trois villages au nord de l'AONB par extension du mécanisme des « conservation areas » (Marie Méténier, 2018).

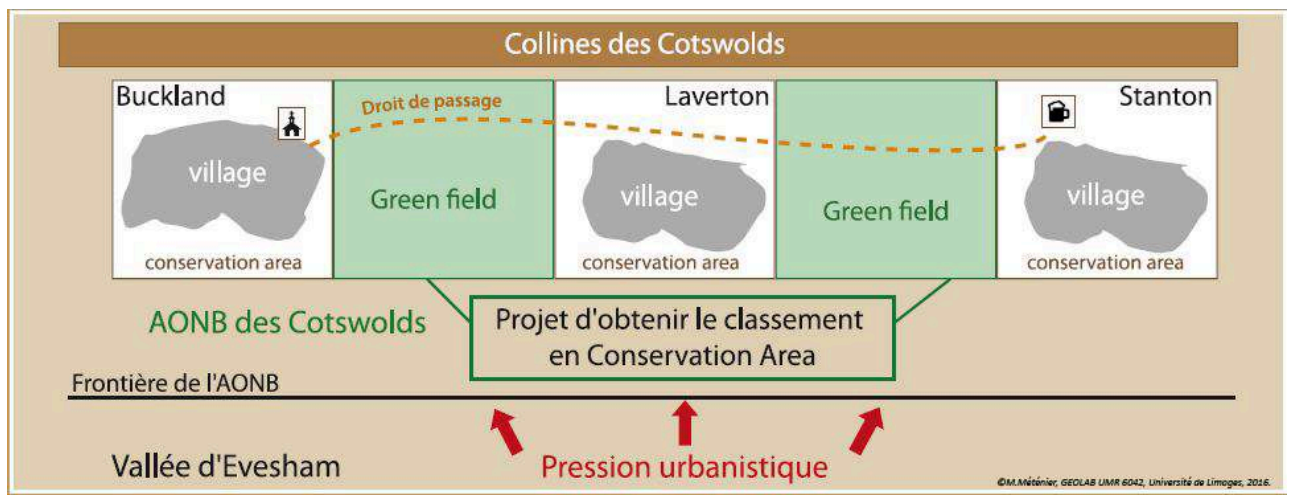

Que ce soit pour se balader, pour se rendre à l'église ou dans le pub du village de Stanton, ces sentiers sont quotidiennement empruntés par les villageois qui considèrent ces champs comme de réelles aménités environnementales qui contribuent à renforcer le sentiment d'appartenance à une communauté. Compte tenu des pressions accrues qui s'exercent sur l'AONB des Cotswolds et qui ont en outre été restituées dans un rapport récent par la CPRE (Campaign to Protect Rural England), l'élu souhaite fusionner les trois conservation areas des trois villages en intégrant les champs qui les séparent afin de les rendre définitivement inconstructibles. En effet, les règles qui s'appliquent dans les conservation areas sont extrêmement strictes et peuvent s'appliquer là où le mécanisme de protection AONB est souvent inefficace pour empêcher les nouveaux projets de construction.

\section{Sauver la vallée de Slad : un contentieux original et potentiellement reproductible.}

Comme le souligne D.Delaney (2009), les affaires judiciaires constituent des angles d'analyses pertinents pour comprendre le rôle joué par le droit dans le maintien et la reproduction des espaces sociaux. Pour ce faire, aller sur le terrain, réaliser des entretiens et identifier directement in situ les conflits juridiques s'inscrit dans la démarche géo-légale évoquée en introduction. Un conflit autour d'un projet de constructions nouvelles s'incarne toujours spatialement et les étapes juridiques du conflit tout comme les arguments avancés par les différentes parties devant le juge révèlent les jeux d'acteurs au sein de l'espace social. Dès lors, mobiliser une méthodologie juridique permet de présenter de manière rigoureuse les étapes d'un conflit juridique en détaillant successivement les faits, les parties au litige, les étapes de la procédure et enfin la solution du litige rendue par le juge. En l'espèce, les frontières ouest de l'AONB des Cotswolds ont fait l'objet d'un contentieux opposant les habitants de la vallée de Slad à un promoteur immobilier. Ce dernier avait déposé une demande d'autorisation de construction auprès de l'autorité locale compétente, à savoir le District de Stroud, dans le but d'implanter un ensemble de constructions neuves dans la continuité urbanistique de la ville de Stroud. L'emprise spatiale du projet (figure 4) portait sur des parcelles agricoles et naturelles situées à la limite extérieure de l'AONB des Cotswolds, mais qui empiétait néanmoins sur la vallée de Slad. 
Figure 4 : Localisation du projet de construction objet du litige entre un promoteur immobilier, les habitants de la vallée de Slad et les associations de protection de l'environnement (Marie Méténier, 2018).

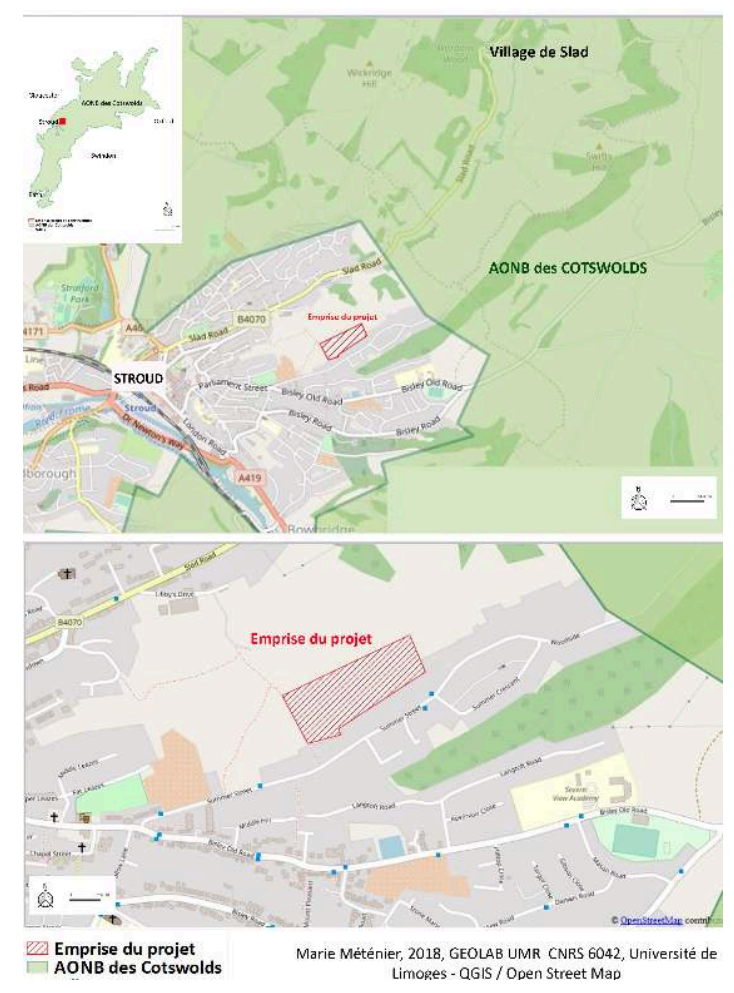

Rapidement, les habitants de la vallée de Slad se sont opposés à ce projet et un groupe local a été constitué sous le nom "Save Slad Valley». Comme souvent, les groupes de pression locaux prennent le nom de l'espace qu'ils souhaitent protéger. D'un point de vue géographique, cela présuppose que les raisons pour lesquelles le groupe de pression local est institué soient partagées par tous les habitants de cet espace. Néanmoins certains semblent tirer plus profit que d'autres de la protection environnementale, ce qui génère et achève d'ancrer des inégalités entre les populations aisées et privilégiées et celles défavorisées (Lowe, $1978: 42$ ). Le groupe constitué pour sauver la vallée de Slad a été soutenu par des associations comme la CPRE (Campaign for the Protection of Rural England). Elle milite depuis sa création en 1926 contre l'étalement urbain et l'artificialisation des parcelles agricoles et naturelles. Il n'est pas surprenant que les habitants interrogés soient pour la majorité des adhérents de cette association (Salomon, Carvin, 2006). D'autres associations comme le Gloucestershire Wildlife Trust ont également été parties au litige pour s'opposer aux dommages causés à la biodiversité de la vallée de Slad. Les entretiens réalisés avec les habitants, notamment ceux du village de Slad révèlent des profils similaires : des ménages aisés, propriétaires, qui travaillent soit de chez eux pour des compagnies d'assurance, des banques ou qui exercent leur activité à Londres (la capitale n'est qu'à $1 \mathrm{~h} 30$ en train de la gare de Stroud). La défense des espaces protégés rencontre donc ici les sensibilités environnementales partagées collectivement par des gentrifieurs à l'image de ceux que P.Cloke et D.Parsons avaient identifiés au début des années 1980. Cependant, il s'agit en l'espèce de relativiser la tendance NYMBISTE des parties prenantes à ce conflit (Garcier, 2009), car ce litige s'inscrit plus dans l'opposition ancrée entre des promoteurs immobiliers et les environnementalistes plutôt que dans des attitudes égoïstes (Abram, Murdoch, 2003). 
Les premiers ont identifié comme une opportunité financière la forte demande de logement et la localisation idéale des parcelles situées en contrebas de la vallée de Slad, dans la continuité urbaine de la ville de Stroud pour des ménages aisés (seul un faible pourcentage de logements financièrement accessibles aux revenus modestes est prévu conformément aux quotas fixés par les autorités locales). De leur côté, les habitants de la vallée et les associations de protection de l'environnement, s'ils semblent conscients dans leurs discours de la nécessité de construire des logements abordables pour les foyers modestes, ont rejeté massivement ce projet qui porterait atteinte à un environnement naturel riche qui constitue un corridor écologique.

"Le promoteur a argumenté devant le juge que son projet n'aurait aucun impact sur l'AONB puisque ce projet se situe à l'extérieur du périmètre de protection. Le promoteur a dit que comme ce n'était pas dans l'AONB, cela n'avait pas d'importance si son projet empiétait sur la vallée de Slad. Mais on a argumenté contre, ce n'est pas parce que le projet n'est pas dans l'AONB que l'impact visuel de celui-ci ne peut pas être perceptible depuis l'AONB. Donc même si ça ne fait pas partie de l'AONB, il faut que ce soit considéré comme différemment d'un point de vue juridique. L'inspecteur du planning nous a entendus, c'est pour ça qu'on a gagné l'affaire ». (Entretien n ${ }^{\circ} 77$, homme, 47 ans, marié, sans enfants, mai 2016)

21 En effet, l'inspecteur du planning, saisi en appel, a rendu sa décision le 22 juillet 2014 après s'être déplacé à plusieurs reprises sur le site en question comme la procédure dans un système de common law le prévoit. En l'espèce, deux procédures d'appel ont été initiées conformément aux dispositions de la section 78 du Town and Country Planning Act de 1990 par le promoteur dans le but d'obtenir l'annulation de deux décisions de refus du District de Stroud, la première datant du 24 avril 2013. Lors de son introduction, le projet de construction proposé par le promoteur portait alors sur un ensemble de constructions de 140 logements d'habitation sur 15 hectares de parcelles agricoles et naturelles. Le projet a été refusé une première fois par l'autorité compétente. En novembre 2013, une nouvelle demande d'autorisation de construire a été déposée par le promoteur (après révision de son projet comprenant 112 logements sur trois parcelles et la création d'un country parc) auprès du District de Stroud qui, une nouvelle fois, a rejeté la demande en février 2014. L'appel formé par le promoteur portait sur les deux décisions de refus, une pratique courante depuis l'arrêt Bernard Wheatcroft LtD $v$ Secretary of State for the Environment de 1982.

L'inspecteur du planning a donc eu à juger en appel de différents motifs présentés par les parties opposées au projet. Ayant été déterminant, un des motifs de refus portait sur l'impact paysager sur la vallée de Slad du projet et sur son insertion entre la limite urbaine de la ville de Stroud et la limite administrative de l'AONB des Cotswolds. En effet, cette dernière ne se situait qu'à 110 mètres du projet et cette partie de la vallée avait été décrite dans le "Cotswolds Landscape Character Assessment » de 2004. Dans sa décision, l'inspecteur souligne précisément que le projet se situe à l'extérieur de l'AONB et qu'il ne bénéficie d'aucune protection au titre du droit de l'environnement. Néanmoins, les arguments avancés par les habitants et les associations de protection de l'environnement contre ce projet ont été validés à deux reprises par l'inspecteur. Tout d'abord, ces premiers soulignaient que la vallée de Slad était attractive grâce aux écrits d'un auteur anglais célèbre, Laurie Lee, et plus particulièrement à travers son ouvrage « Cider with Rosie ". Comme le souligne l'inspecteur (point 22 de la décision), bien que l'auteur ne fasse pas de référence claire dans ses écrits aux parcelles concernées par le projet de construction, il est admis que ces parcelles font partie intégrante de la vallée 
de Slad. L'inspecteur retient la corrélation évidente entre les aménités environnementales présentées par la vallée de Slad dans son entièreté, l'ouvrage de Laurie Lee et l'attractivité de cet espace. Si l'inspecteur n'y fait pas référence directement pour motiver sa décision, les entretiens et les parcours commentés réalisés avec les habitants ont permis de révéler qu'un sentier de nature appelé «Laurie Lee Wildlife Way " avait été créé par le Gloucestershire Wildlife Trust, en partenariat avec les autorités locales et les habitants du village de Slad pour valoriser le patrimoine naturel et culturel de la vallée de Slad (figure 5).

Figure 5 : Mise en image du contentieux : quand les marqueurs spatiaux rejoignent les arguments juridiques (Marie Méténier, 2017).

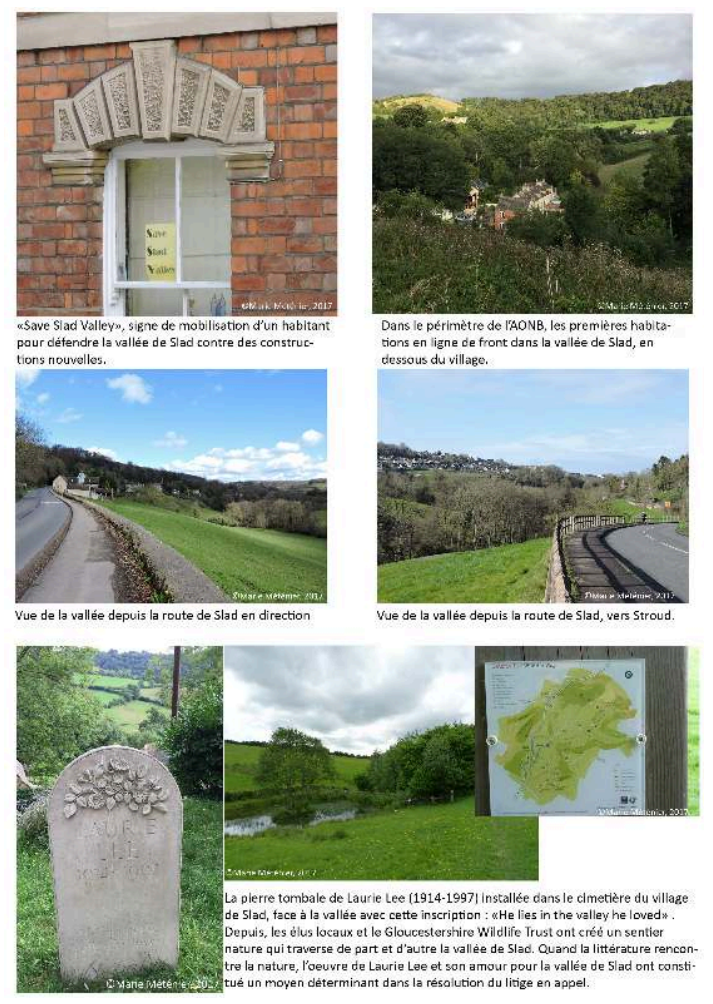

Dans sa décision d'appel, l'inspecteur est également venu considérer les arguments de refus spécifiquement avancés par le district de Stroud qui invoquait notamment la nonconformité du projet aux dispositions de la règlementation NE8 Local Plan réalisé pour la ville de Stroud. La disposition NE8 est relative à la réalisation de projets de construction dans et autour du périmètre de l'AONB et à l'impact paysager d'un projet sur et depuis l'AONB. Conformément à cette disposition, le projet a été rejeté à la fois par l'autorité locale et par l'inspecteur du planning. Si la décision de rejet de l'appel par l'inspecteur du planning a permis de rassurer les habitants de la vallée de Slad, ces derniers ont souligné à postériori dans leurs discours leurs inquiétudes concernant les pressions futures et les menaces qui pesaient sur « leur vallée ».

« Depuis le dernier appel que nous avons remporté, le même promoteur est revenu avec un projet moins important, mais le town council a rejeté une nouvelle fois sa demande. Le promoteur essayait de dire que les règles devaient être interprétées de manière stricte et que si le projet n'était pas dans l'AONB alors il devait être autorisé. Nous avons dit que ce n'est pas parce qu'une parcelle naturelle ou agricole n'est pas dans l'AONB qu'elle n'a pas de valeur environnementale. 
Ceux qui ont créé les frontières de l'AONB n'ont juste pas réfléchi 40 ans plus tôt ». (Entretien $\mathrm{n}^{\circ} 77$, homme, 47 ans, marié, sans enfants, mai 2016)

Depuis 2018, un financement participatif est désormais ouvert pour tenter de racheter collectivement les parcelles de la vallée de Slad qui ne se situe pas dans le périmètre de l'AONB afin de les rendre inconstructibles à perpétuité et de les gérer en communauté. Intitulé "I want to save the field forever ", cette initiative n'est certainement pas isolée à la seule vallée de Slad et le précédent créé en l'espèce par la décision d'appel peut laisser supposer que ce contentieux sera potentiellement reproductible à la frontière d'autres espaces protégés anglais.

\section{Conclusion :}

La géographie du droit apparaît comme une entrée particulièrement riche pour analyser les formes et les conséquences de la gentrification rurale dans un espace protégé. En élargissant le champ d'application d'instruments juridiques ou en renforçant certaines contraintes règlementaires, les gentrifieurs participent à de nouvelles productions spatiales, que ce soit pour sauvegarder leur cadre de vie ou pour sécuriser la valeur marchande de leur patrimoine immobilier. Cette démarche permet d'analyser différentes échelles d'instrumentalisations des documents d'urbanisme et des mécanismes de protection de l'environnement qui conduisent à différentes formes de confiscation et de contrôle des espaces protégés (Blomley, 2001, p283). Ces instrumentalisations à l'interface géo-légale contribuent à renforcer les logiques d'appropriation, de domination et donc à reproduire les inégalités sociales (Melé, 2009). Comme le souligne S. Depraz (2005), chaque espace "naturel» protégé est une problématique en soi. Par conséquent, on peut légitimement supposer que les modes d'appropriations et d'instrumentalisations $d u$ droit résultant $d u$ processus de gentrification rurale peuvent revêtir des formes inédites selon les types d'espaces protégés étudiés. Dès lors, une étude plus approfondie de la gentrification rurale dans les parcs nationaux anglais permettrait de révéler d'autres formes d'instrumentalisation et de dominations spatiales par le droit. En effet, depuis 1995, les parcs nationaux anglais sont les autorités compétentes en matière d'urbanisme et d'aménagement pour la superficie des parcs. Cette compétence en matière d'urbanisme justifie certainement les discussions récentes autour du projet de création d'un parc national des Cotswolds pour pallier aux lacunes du mécanisme AONB. De plus, la présomption d'une gentrification rurale plus soutenue dans les parcs nationaux se justifie dans la mesure où elle avait, dès les origines du concept, été soulevée par les chercheurs britanniques (Parsons, 1980 ; Cloke, 1983). L'investissement des sphères du planning system par différents acteurs lors de l'élaboration des documents d'urbanisme constitue un axe de recherche pertinent dans la mesure où il s'agit d'un levier d'action pour contrôler, en amont, l'espace à l'échelle locale (Little, 1987 ; Murdoch et Marsden, 2013). Enfin, les recherches effectuées sur le processus de gentrification rurale se sont souvent concentrées sur les "gentrifieurs", mais très peu sur les populations « locales", marginalisées dans la littérature (Dirksmeier, 2008). Or, comme le souligne David Delaney (2009), la géographie du droit permet aussi de s'intéresser à ceux qui ont été « victimes » de ségrégations ou d'expulsions. Dès lors la mise en place de solutions par différents acteurs pour résister à la gentrification rurale constitue une piste de recherche pertinente. 


\section{BIBLIOGRAPHIE}

Abrams, J. B., Gosnell, H., Gill, N. J., Klepeis, P. J., 2012, « Re-creating the rural, reconstructing nature: An international literature review of the environmental implications of amenity migration. » Conservation and Society, vol.10, n³, p.270-284.

Bailoni M., 2012, « Aménager un espace idéalisé : identité et conflits dans la campagne anglaise », Revue Géographique de l'Est [En ligne], vol. 52 / 3-4 | 2012, mis en ligne le 01 juillet 2013, consulté le 14 septembre 2016. URL : http://rge.revues.org/3739

Blomley N., 2001, « Introduction » in D. Delaney, \& R. T. Ford (Eds.), The Legal Geographies Reader: Law, Power and Space, Oxford, Blackwell Publishers, p.252-255.

Blomley N., 2004, « The boundaries of property: lessons from Beatrix Potter », The Canadian Geographer/Le Géographe canadien, vol.48, n², p.91-100.

Blunden J., Curry N., 1990, A people's charter? Forty years of the National Parks and Access to the Countryside Act 1949, London, HMSO Publications Centre, 299 p.

City C., 2009, «La géographie du droit, l'eau et l'environnement », in Forest P., 2009, Géographie du droit: épistémologie, développement et perspectives, Québec, Presses de l'Université Laval, p.157-176.

Clark E., 2005, « The order and simplicity of gentrification: a political challenge », In Gentrification in a global context: the new urban colonialism, London, Routledge, p.261-269.

Cloke P., 1979, Key Settlements in Rural Areas, London, Methuen, 259 p.

Cloke P., 1983, An Introduction To Rural Settlement Planning, London, Methuen, 380 p.

Cloke, P., Phillips, M., Thrift, N., 1995, « The new middle classes and the social constructs of rural living. ", Social change and the middle classes, p.220-238.

Cognard F., 2010, Migrations d'agrément et nouveaux habitants dans les moyennes montagnes françaises : de la recomposition sociale au développement territorial. L'exemple du Diois, du Morvan et du Séronnais, thèse, Université Blaise Pascal - Clermont-Ferrand II, 528 p.

Delaney D., 2003, Law and nature, Cambridge, Cambridge University Press, 440 p.

Delaney D., 2009, «Le juridique, le spatial et la pragmatique de la construction de la réalité », in Forest P., 2009, Géographie du droit: épistémologie, développement et perspectives, Québec, Presses de l'Université Laval, p.117-137.

Depraz, S., 2005, Recompositions territoriales, développement rural et protection de la nature dans les campagnes d'Europe centrale post-socialiste, 2005, thèse, Université Paul Valéry-Montpellier III, 525 p.

Depraz S., 2008, Géographie des espaces naturels protégés, Armand Colin, Paris, 320 p.

Desponds D., 2007, «Les impacts d'un parc naturel régional (PNR) sur les évolutions sociodémographiques de son espace rural : le cas du Vexin français ", Norois. Environnement, aménagement, société (202): 47-60.

Dirksmeier, P., 2008, « Strife in the rural idyll? The relationship between autochthons and inmigrants in scenic regions of South Bavaria ", Erdkunde, p.159-171.

Forest P., 2009, Géographie du droit: épistémologie, développement et perspectives, Québec, Presses de l'Université Laval, 286 p. 
Glorioso R., Moss L., 2012, « Origines et développement du concept de migration d'agrément », In Les migrations d'agrément: du tourisme à l'habiter, N.Martin, P.Bourdeau, J.F. Daller (Eds.), p.37-55.

Holdaway E., Smart G., 1999, Landscapes at Risk? The Future for Areas of Outstanding Natural Beauty in England and Wales, London, Taylor \& Francis, $242 \mathrm{p}$.

Little J., 1987, « Rural gentrification and the influence of local-level planning », in Cloke P., Rural planning: policy into action, London, Harper and Row, p.185-199.

Lowe, P. D., 1977, « Amenity and equity: a review of local environmental pressure groups in Britain », Environment and Planning A, vol.9, n²1, p.35-58.

Lowe, P., Murdoch, J., 2003, « Mediating the 'national'and the 'local'in the environmental policy process: a case study of the CPRE », Environment and Planning C: Government and Policy, vol.21, $\mathrm{n}^{\circ} 5$, p.761-778.

MacEwen A., \& MacEwen M., 1982, National parks: conservation or cosmetics?, London, George Allen and Unwin, $314 \mathrm{p}$.

Marsden T., Murdoch J., Lowe P., Munton RC., Flynn A., 1996, Constructing The Countryside, London, Routledge, $220 \mathrm{p}$.

Murdoch J., Lowe P., 2003, « The preservationist paradox : modernism, environmentalism and the politics of spatial division », Transaction of the Institute of British Geographers, n²8, p.318-332.

Murdoch, J., Marsden, T., 2013, Reconstituting rurality, London, Routledge, 272p.

Nelson P., Oberg A., Nelson L., 2011, « The global rural: Gentrification and linked migration in the rural USA », Progress in Human Geography, vol.35, n4, p.441-459.

Newby, H., 1980, Green and pleasant land? Social change in rural England, London, Penguin Books Ltd, $301 \mathrm{p}$.

Parsons, D., 1979, A geographical examination of the twentieth century theory and practice of selected village development in England, thèse, Université de Nottingham, 870 p.

Parsons D., 1980, Rural gentrification: the influence of rural settlement planning policies, University of Sussex : Brighton.

Phillips M., 1993, « Rural gentrification and the process of class colonisation », Journal of Rural Studies, vol.9, n², p.123-140.

Phillips M., 2010, "Counterurbanisation and rural gentrification : an exploration of the terms ", Population, Space and Place, vol.16, n%, p.539-558. DOI:10.1002/psp.570.

Platt R.H., 2004, Land Use and Society, Revised Edition: Geography, Law, and Public Policy, London, Island Press, $544 \mathrm{p}$.

Preece, R. A., 1981, Patterns of development control in the Cotswolds Area of Outstanding Natural Beauty (No. 27), School of Geography, University of Oxford.

Richard, F., 2009, «La gentrification des «espaces naturels» en Angleterre: après le front écologique, l'occupation? L'exemple du Lake District et de ses environs. », L'Espace Politique. Revue en ligne de géographie politique et de géopolitique, (9).

Richard F., Dellier J., Tommasi G., 2014, « Migration, environnement et gentrification rurale en Montagne limousine ", Journal of Alpine Research/ Revue de géographie alpine (102-4).

Richard, F., 2017, La gentrification rurale, de l'observation du fait géographique à la circulation du concept. Habilitation à diriger des recherches, vol.1, $227 \mathrm{p}$. 
Schmied D., 2002, What price peace and quiet? : rural gentrification and affordable housing; the exemple of Cotswolds District, South West of England, Bayrouth, Naturwiss, Ges, 196 p.

Smith D., 1998, The revitalisation of the Hebden Bridge District: gentrified Pennine rurality, thèse, Université de Leeds, 348 p.

Smith D., Philips DA., 2001, « Socio-cultural representations of greentrified Pennine rurality », Journal of Rural Studies, vol.17, n², p.457-469.

Urry J., 1995, « A middle-class countryside? », In Social change and the middle classes, Butler T., Savage M., (Eds.), UCL Press: London, p. 205-219.

Woods M., 2007, « Engaging the global countryside: globalization, hybridity and the reconstitution of rural place ", Progress in Human Geography , vol.31, n4, p.485-507.

Woods, M., 2017, « Politics and protest in the contemporary countryside ", In Geographies of rural cultures and societies, London, Routledge, p.103-125.

\section{NOTES}

1. http://www.cotswoldsaonb.org.uk/userfiles/file/meetings-2016/board-24-mar-16/8bii-ls-andg-7-high-wold.pdf

2. Not In My BackYard

\section{RÉSUMÉS}

Les espaces protégés anglais sont sujets à des recompositions sociales et démographiques pouvant s'analyser au prisme du processus de la gentrification rurale. Si les interactions entre les espaces ruraux et la gentrification rurale ont été largement traitées par la littérature scientifique, le lien avec les espaces protégés n'a été que peu exploré. Pourtant, cet axe de recherche constitue une opportunité de croiser les deux disciplines que sont le droit et la géographie dans une démarche interdisciplinaire propre à la géographie du droit. Les espaces protégés anglais cristallisent des conflits d'appropriations et d'instrumentalisations du droit de l'urbanisme et de l'environnement par des gentrifieurs soucieux de contrôler et de protéger «leurs » espaces. Cette volonté de contrôle des espaces protégés s'étend parfois au-delà des périmètres de protection et se traduit juridiquement par des gentrifieurs qui s'engagent dans des procédures contentieuses.

English gentrified protected areas are subject to socio-economic changes which can be explored through the rural gentrification process. If interactions between English rural spaces and rural gentrification have been widely explored by the literature, the link with the protected areas has been neglected. And yet, this topic is an opportunity to cross two subjects which are Geography and Law in an interdisciplinary approach inherent of the Legal Geography. Protected areas in England form conflicts of seizing and uses of the planning system and environmental law by gentrifiers seeking to control and protect "their" spaces. This wish to control protected areas extends sometimes beyond their administrative boundaries and lead to contentious issues and planning appeals. 
Englisch gentrifizierte Schutzgebiete unterliegen sozioökonomischen Veränderungen, die durch den ländlichen Gentrifizierungsprozess untersucht werden können. Wenn Wechselwirkungen zwischen englischen ländlichen Räumen und ländlicher Gentrifizierung in der Literatur ausführlich untersucht wurden, wurde die Verbindung zu den Schutzgebieten vernachlässigt. Dieses Thema ist jedoch eine Gelegenheit, zwei Themen, Geografie und Recht, in einem interdisziplinären Ansatz zu überqueren, der der Rechtsgeographie innewohnt. Geschützte Gebiete in England führen zu Konflikten bei der Ausnutzung und Nutzung des Planungssystems und des Umweltrechts durch Gentrifier, die versuchen, "ihre" Räume zu kontrollieren und zu schützen. Dieser Wunsch, geschützte Gebiete $\mathrm{zu}$ kontrollieren, reicht manchmal über die administrativen Grenzen hinaus und führt zu umstrittenen Fragen

\section{INDEX}

Mots-clés : gentrification rurale, géographie du droit, espaces protégés, Angleterre.

Schlüsselwörter : Ländlichen Gentrifizierung, Gentrificatie, Landschaftsschutz, legaal geografie Keywords : rural gentrification, legal geography, protected areas, England.

\section{AUTEUR}

\section{MARIE MÉTÉNIER}

Doctorante - GEOLAB UMR CNRS 6042 - Université de Limoges- marie.metenier@unilim.fr 